\title{
A variant near MTNR1B is associated with increased fasting plasma glucose levels and type 2 diabetes risk
}

\author{
Nabila Bouatia-Naji ${ }^{1,26}$, Amélie Bonnefond ${ }^{1,26}$, Christine Cavalcanti-Proença ${ }^{1}$, Thomas Sparsø ${ }^{2}$, \\ Johan Holmkvist $^{2}$, Marion Marchand ${ }^{1}$, Jérôme Delplanque ${ }^{1}$, Stéphane Lobbens ${ }^{1}$, Ghislain Rocheleau ${ }^{3-5}$, \\ Emmanuelle Durand $^{1}$, Franck De Graeve ${ }^{1}$, Jean-Claude Chèvre ${ }^{1}$, Knut Borch-Johnsen ${ }^{2,6}$, \\ Anna-Liisa Hartikainen ${ }^{7}$, Aimo Ruokonen ${ }^{8}$, Jean Tichet ${ }^{9}$, Michel Marre ${ }^{10}$, Jacques Weill ${ }^{11}$, Barbara Heude ${ }^{12}$, \\ Maithé Tauber ${ }^{13}$, Katleen Lemaire ${ }^{14}$, Frans Schuit ${ }^{14}$, Paul Elliott ${ }^{15}$, Torben Jørgensen ${ }^{16,17}$, \\ Guillaume Charpentier ${ }^{18}$, Samy Hadjadj ${ }^{19}$, Stéphane Cauchi ${ }^{1}$, Martine Vaxillaire ${ }^{1}$, Robert Sladek ${ }^{3,4}$, \\ Sophie Visvikis-Siest ${ }^{20}$, Beverley Balkau ${ }^{12}$, Claire Lévy-Marchal ${ }^{21}$, François Pattou ${ }^{22}$, David Meyre ${ }^{1}$, \\ Alexandra I F Blakemore ${ }^{23}$, Marjo-Riita Jarvelin ${ }^{15,24}$, Andrew J Walley ${ }^{23}$, Torben Hansen ${ }^{2,25}$, Christian Dina ${ }^{1}$, \\ Oluf Pedersen $^{2,6,17}$ \& Philippe Froguel ${ }^{1,23}$
}

In genome-wide association (GWA) data from 2,151 nondiabetic French subjects, we identified rs1387153, near MTNR1B (which encodes the melatonin receptor 2 (MT2)), as a modulator of fasting plasma glucose (FPG; $P=1.3 \times 1^{-7}$ ). In European populations, the rs1387153 $\mathrm{T}$ allele is associated with increased FPG $\left(\beta=0.06 \mathrm{mmol} / \mathrm{l}, P=7.6 \times 10^{-29}\right.$, $N=16,094)$, type 2 diabetes (T2D) risk (odds ratio (OR) = $1.15,95 \% \mathrm{Cl}=1.08-1.22, P=6.3 \times 10^{-5}$, cases $N=6,332$ ) and risk of developing hyperglycemia or diabetes over a 9-year period (hazard ratio $(\mathrm{HR})=1.20,95 \% \mathrm{Cl}=1.06-1.36$, $P=0.005$, incident cases $N=515$ ). RT-PCR analyses confirm the presence of MT2 transcripts in neural tissues and show MT2 expression in human pancreatic islets and beta cells. Our data suggest a possible link between circadian rhythm regulation and glucose homeostasis through the melatonin signaling pathway.

Elevated fasting plasma glucose (FPG) levels within the range specified by the American Diabetes Association ${ }^{1,2}$ are an independent risk factor for type 2 diabetes (T2D) ${ }^{3}$ and are associated with increased risk of mortality in prospective studies ${ }^{4}$. We have previously identified a common genetic variant in the G6PC2 (glucose-6-phosphatase catalytic unit 2) explaining $\sim 1 \%$ of the variance of FPG at the population level, although not increasing the risk of $\mathrm{T}^{2} \mathrm{D}^{5}$. Following this, we aimed to identify new genetic variation involved in glucose homeostasis using GWA data from 2,151 nondiabetic French individuals with European ancestry.

We carried out a GWA meta-analysis for FPG using data from four independent populations, including 715 lean adults, 614 lean children, 247 obese adults and 575 obese children (Supplementary Table 1, $N=2,151)$. In addition to G6PC2 $\left(P=4.1 \times 10^{-7}\right)$, we identified two SNPs that reach genome-wide significance for association with FPG: rs4240702 on chromosome $9\left(P=8.0 \times 10^{-9}\right.$, adjusted genome-wide $P=0.002$ ) and $\mathrm{rs} 1387153$ on chromosome 11 $\left(P=1.3 \times 10^{-7}\right.$, adjusted genome-wide $\left.P=0.04\right)$. A first replication analysis in 3,886 nondiabetic individuals from the French DESIR population (a longitudinal general population cohort with mean

${ }^{1}$ CNRS-UMR-8090, Institute of Biology and Lille 2 University, Pasteur Institute, F-59019 Lille, France. ${ }^{2}$ Steno Diabetes Center, Niels Steensens Vej 1, NLC2.13, DK-2820 Gentofte, Denmark. ${ }^{3}$ Department of Human Genetics, Faculty of Medicine, McGill University Montreal H3H 1P3, Canada. ${ }^{4}$ Genome Quebec Innovation Centre, Montreal H3A 1A4, Canada. ${ }^{5}$ Prognomix, Montreal, H1Y 3 L1 Canada. ${ }^{6}$ Faculty of Health Science, University of Aarhus, DK-8000 Aarhus C, Denmark. ${ }^{7}$ Department of Clinical Sciences/ Obstetrics and Gynecology, University of Oulu, FIN- 90014, Finland. ${ }^{8}$ Department of Clinical Sciences/ Clinical Chemistry, University of Oulu, FIN- 90014 University of Oulu, Finland. ${ }^{9}$ Institut inter-régional pour la santé (IRSA), F-37521 La Riche, France. ${ }^{10}$ Department of Endocrinology, Diabetology and Nutrition, Bichat-Claude Bernard University Hospital, Assistance Publique des Hôpitaux de Paris, F-75018 Paris, France, and INSERM U695, Université Paris 7 , F-75870 Paris, France. ${ }^{11}$ Pediatric Endocrine Unit, Jeanne de Flandre Hospital, F-59800 Lille, France. ${ }^{12}$ INSERM U780, Villejuif, F-94807; University Paris-Sud, Orsay, F-91405, France. ${ }^{13}$ INSERM U563, Children's Hospital, CHU F-31059 Toulouse, France. ${ }^{14}$ Gene Expression unit, Department Molecular Cell Biology Katholieke Universiteit B-3000 Leuven, Belgium. ${ }^{15}$ Department of Epidemiology and Public Health, Imperial College London, W2 1PG London, UK. ${ }^{16}$ Research Centre for Prevention and Health, Glostrup University Hospital, DK-2600 Denmark. ${ }^{17}$ Faculty of Health Science, University of Copenhagen, DK-2200 Copenhagen, Denmark. ${ }^{18}$ Endocrinology-Diabetology Unit, Corbeil-Essonnes Hospital, Essonnes, F-91108 France. ${ }^{19}$ Centre hospitalier universitaire de Poitiers, Endocrinologie Diabetologie, CIC INSERM 0802, INSERM U927, Université de Poitiers, UFR Médecine Pharmacie, F-86034 Poitiers, France. 20INSERM "Cardiovascular Genetics" team, CIC 9501, F-54000 Nancy, France. ${ }^{21}$ INSERM, Unité 690, Robert Debré hospital, Paris, F- 75019, France, Paris Diderot University, Paris, F-75005, France. 22INSERM U859, CHRU de Lille, Université Lille-Nord de France, F-59045 Lille, France. ${ }^{23}$ Genomic Medicine, Hammersmith Hospital, Imperial College London, W12 0NN, London, UK. ${ }^{24}$ Institute of Health Sciences, University of Oulu, Finland, Department of Child and Adolescent Health, National Public Health Institute, Finland, Biocenter Oulu, University of Oulu, FIN- 90014 Oulu, Finland. ${ }^{25}$ Faculty of Health Science, University of Southern Denmark, Odense, DK-5230 Denmark.

26These authors equally contributed to this work. Correspondence should be addressed to P.F. (p.froguel@imperial.ac.uk). 
Table 1 Association of rs1387153 with fasting plasma glucose levels in nondiabetic individuals from a genome-wide association meta-analysis study and replication studies

Mean fasting plasma glucose $(\mathrm{mmol} / \mathrm{l})$ by genotype

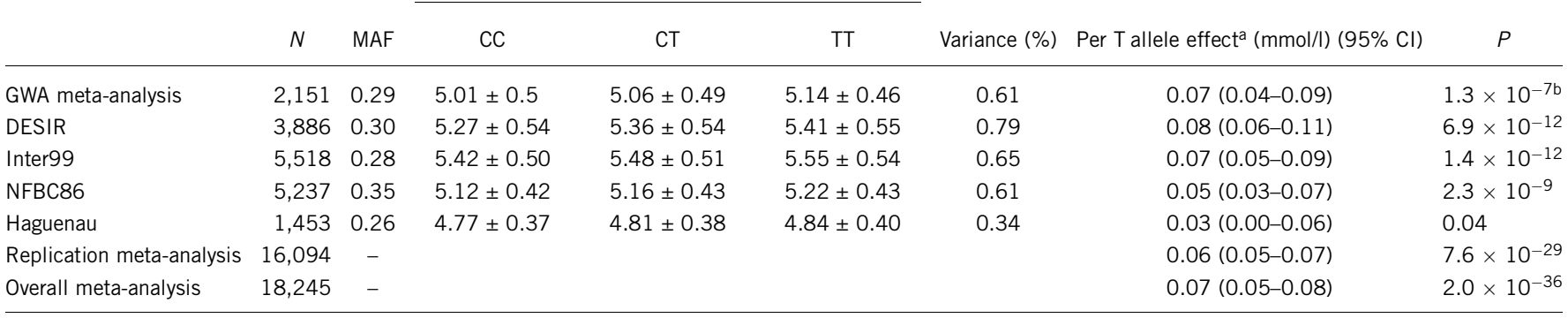

Association was tested using an additive model, adjusted for age, sex and BMI. All individuals were nondiabetic (FPG $<7$ mmol/I without glucose lowering agents). DESIR excluded the 715 participants that were part of the GWA meta-analysis. The replication meta-analysis excluded GWA meta-analysis.

aPer-T allele effect size is the regression coefficient $\beta$. ${ }^{\text {b} A d j u s t e d ~ g e n o m e-w i d e ~} P$ value is 0.04 .

age $=46.5 \pm 10.2$ years $)$ confirmed the association of the rs1387153 T allele with increased FPG $(\beta=0.08 \mathrm{mmol} / \mathrm{l} 95 \% \mathrm{CI}=0.06-0.11$, $P=6.9 \times 10^{-12}$; Table 1), but did not replicate the association of FPG with rs4240702 $(P=0.21)$. Analysis in 5,518 middle-aged nondiabetic individuals (mean age $=45.9 \pm 7.8$ years) from the Danish population-based study Inter99 provides further support for the effect of rs1387153 on FPG ( $\beta=0.07 \mathrm{mmol} / \mathrm{l}, 95 \%$ $\mathrm{CI}=0.05-0.09), P=1.4 \times 10^{-12}$; Table 1). SNP rs1387153 also associates with increased FPG both in 5,237 young nondiabetic individuals (age at examination $=16$ years) from the Northern Finland Birth Cohort 86 (NFBC86) $(\beta=0.05 \mathrm{mmol} / \mathrm{l}, 95 \% \mathrm{CI}=0.03-0.07$, $\left.P=2.3 \times 10^{-9}\right)$ and in 1,453 young adults from the French Haguenau cohort $($ mean age $=22.1 \pm 3.9$ years; $\beta=0.03 \mathrm{mmol} / \mathrm{l} ; 95 \%$ $\mathrm{CI}=0.00-0.06, P=0.04$; Table 1).

Further analysis of metabolic traits in the nondiabetic participants of the DESIR cohort showed that SNP rs1387153 is associated with an increased fraction of erythrocyte glycosylated hemoglobin (HbAlc) $\left(\beta=0.04 \%, 95 \%\right.$ CI $=0.02-0.06, P=1.7 \times 10^{-4}$; Supplementary Table 2), an indicator of a deteriorated glucose control over the previous two to three months, as well as with decreased basal insulin release as assessed by the homeostasis model of pancreatic beta cell function index HOMA-B $(\beta=-5.82 \%$, 95\% CI $=-7.69-2.96$, $P=3.3 \times 10^{-5}$; Supplementary Table 2), suggesting that the association with increased FPG may result, at least in part, from impaired insulin secretion. In contrast, no association was observed with the homeostasis model of insulin resistance index (HOMA-IR; $P=0.46$; Supplementary Table 2). There was only a trend for an association with body mass index $(P=0.066$; Supplementary Table 2) in the DESIR cohort and GWA case-control analysis for obesity including 1,380 French severely obese and 1,416 normal weight controls did not reveal any association with obesity at this locus ( $P=0.71$ for rs1387153), indicating that the observed effect on FPG is independent of obesity-induced insulin resistance (unpublished data). The effects of rs1387153 on other metabolic traits are shown in Supplementary Table 2 for the DESIR cohort and Supplementary Table 3 for the Inter99 study sample.

We then assessed the contribution of rs1387153 to T2D risk in 6,332 French and Danish diabetic subjects and 9,132 normal glucose French and Danish controls selected from the DESIR cohort and the Inter99 study sample, respectively. We found that the rs1387153 $\mathrm{T}$ allele increases the risk of $\mathrm{T} 2 \mathrm{D}(\mathrm{OR}=1.15,95 \% \mathrm{CI}=1.08-1.22$, $P=6.3 \times 10^{-5}$; Supplementary Table 4), indicating that the effect of rs1387153 on FPG is also observed in the ultimate consequence of elevated FPG, which is T2D, in contrast to the effect of the G6PC2 variant, which was proposed as a genetic determinant for FPG only in general populations ${ }^{5}$. In accord with our findings, data from the DIAGRAM consortium show nominally significant association between rs1387153 and T2D assessed in 4,549 cases and 5,579 controls $(P=0.015)$. Longitudinal analyses of the DESIR cohort demonstrated that carriers of the rs $1387153 \mathrm{~T}$ allele have a $20 \%$ increase in the risk of developing hyperglycemia (defined as FPG $\geq 6.1 \mathrm{mmol} / \mathrm{l}$ or diabetes) over the 9-year follow-up period (hazard ratio $=1.20,95 \%$ $\mathrm{CI}=1.06-1.36, P=0.005)$.

We also analyzed the cumulative effect of rs1387153 and the three previously identified genetic determinants of FPG (G6PC2 rs560887, GCKR rs1260326 (P446L) and GCK rs1799884 (-30G/A) $)^{5}$ in the DESIR cohort and observed an additive effect (Fig. 1). Subjects with three 'high FPG' alleles have a mean FPG of $5.26 \mathrm{mmol} / \mathrm{l}$ (95\% CI $=5.23-5.29)$, which is above the threshold of $94 \mathrm{mg} / \mathrm{dl}$ (5.21 $\mathrm{mmol} / \mathrm{l})$, previously reported to double the risk of T2D (adjusted for age, BMI and triglyceride level) in young healthy individuals ${ }^{3}$. Those carrying six or more high FPG alleles $(4.5 \%$ of the DESIR participants, $N=183$ ) show a mean $0.36 \mathrm{mmol} / \mathrm{l}$ increase

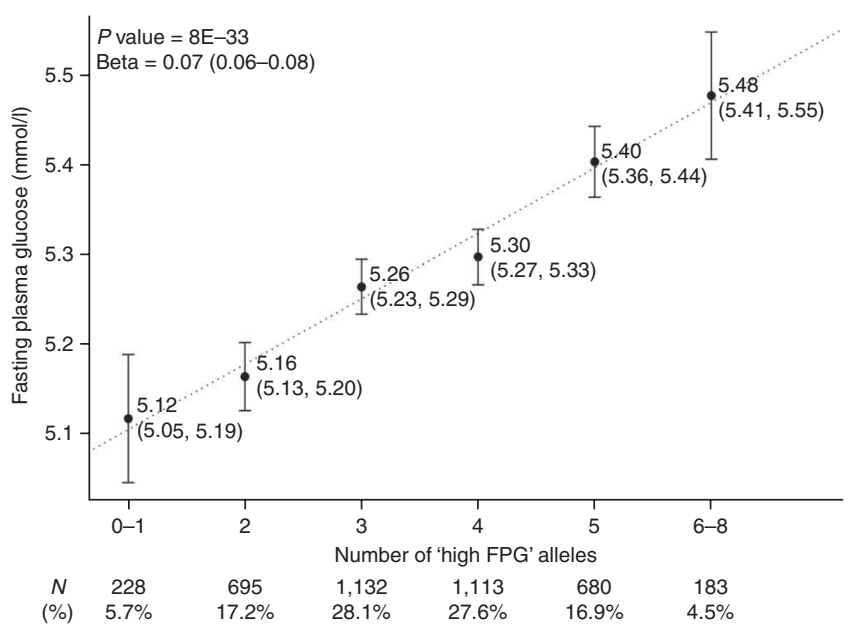

Figure 1 Cumulative effects of MTNR1B rs1387153, G6PC2 rs560887, GCKR rs1260326 (P446L) and GCK rs1799884 (-30G) variants on fasting plasma glucose levels in the DESIR cohort. Data are presented as mean (95\% $\mathrm{Cl}$ ) and the $\beta$ coefficient in the linear regression model (including age, sex and BMI as covariates) corresponds to the increase of fasting plasma glucose (FPG) levels by additional 'high FPG' alleles. The numbers of individuals per category of 'high FPG' allele carriers and the corresponding percentages in the DESIR cohort are shown below the graph. 

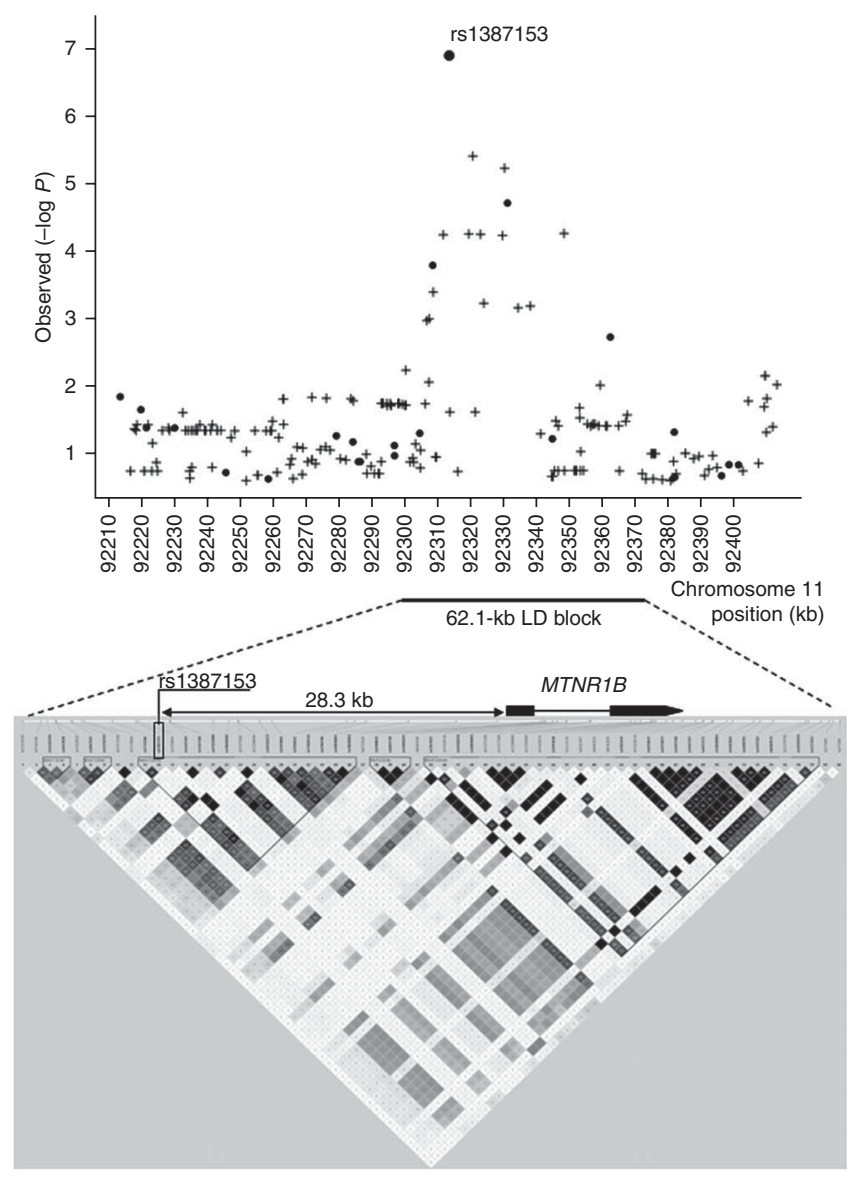

in FPG compared to individuals with zero or one high FPG allele (Fig. 1) and, importantly, $43 \%$ of them $(N=78)$ reach the epidemiological threshold of impaired fasting glucose, $5.6 \mathrm{mmol} / \mathrm{l}$, set by the American Diabetes Association ${ }^{1}$.

SNP rs1387153 maps within a 62.1-kb linkage disequilibrium (LD) block on chromosome 11 and is located in the $5^{\prime}$ region of MTNR1B, encoding the melatonin receptor $1 \mathrm{~B}$ (MT2, Fig. 2). To assess the contribution of putative coding variants in MTNR1B to the association with FPG, we carried out direct sequencing of the two exons (362 amino acids) of MTNR1B in 638 nondiabetic individuals randomly selected from the DESIR population. None of the 14 exonic variants identified (Supplementary Table 5) is in LD with rs1387153 or associates with FPG. SNP rs1387153 is in high LD $\left(r^{2}>0.70\right)$ with seven SNPs within the 62.1-kb LD block that were not genotyped here. Three of them $\left(\right.$ rs10765573 $\left(r^{2}=0.82\right), \operatorname{rs} 7936247\left(r^{2}=0.84\right)$ and rs11020124 $\left.\left(r^{2}=0.94\right)\right)$ are part of a conserved genomic region across
Figure 2 Genomic context and association with fasting plasma glucose of rs1387153 on chromosome 11q21. The upper panel shows the association magnitude $(-\log (P$ value $))$ with fasting plasma glucose of the tag SNPs (dots) and the HapMap CEU data imputed SNPs (plus signs) not directly genotyped around $( \pm 100 \mathrm{~kb}$ ) rs1387153. The lower panel shows the LD structure (defined by pairwise $r^{2}$ ) of the 62.1-kb LD block that includes rs1387153 and MTNR1B between SNPs in the CEU population from HapMap phase II using the Haploview software. MTNR1B gene organization and rs1387153 position and distance from MTNR1B are indicated.

species located in the proximal $5^{\prime}$ region of MTNR1B. One SNP, rs10830963 $\left(r^{2}=0.70\right)$, is located in the only intron of MTNR1B. Imputation of genotypes using the LD structure from HapMap data (CEU population) for SNPs in LD with rs1387153 (and all SNPs $100 \mathrm{~kb}$ upstream and $100 \mathrm{~kb}$ downstream rs1387153 with data available in the HapMap CEU samples) did not identify any SNP showing potentially stronger association with FPG than rs1387153 (Fig. 2). Nonetheless, all SNPs of the LD block including MTNR1B are putative candidates for functionality of the association signal with FPG identified in our study.

Melatonin is a neurohormone that regulates the circadian rhythm by translating photoperiodic information from the eyes to the brain. Melatonin signaling is mainly mediated by two receptors: MT1 and MT2, encoded by MTNR1A and MTNR1B, respectively ${ }^{6}$. The strongest MT2 expression levels are detected in the retina and the suprachiasmatic nucleus (SCN), the circadian rhythm control center ${ }^{6}$. Using RT-PCR on a panel of human tissues, we confirmed the expression of MTNR1B in the retina, the brain and, more specifically, in the diencephalon, including the hypothalamus and the SCN (Fig. 3). MT2-mediated melatonin signaling could indirectly regulate glucose levels and insulin secretion through the brain control center of the circadian clock, as proposed previously ${ }^{7}$. In healthy individuals, insulin secretion follows a circadian rhythm with peaks during the day, in an opposite manner to melatonin circulating levels ${ }^{8}$, and both melatonin secretion and circadian rhythm are impaired in $\mathrm{T}_{2} \mathrm{D}^{9}$. Melatonin is a potent antioxidant agent and has been suggested to directly influence insulin secretion. The MT1 receptor is highly expressed in pancreatic islets, although MT2 expression in islets and beta cells is still elusive ${ }^{10}$. We confirmed the expression of MTNR1B in both islets and sorted beta cells (Fig. 3), supporting a putative direct role of MT2 in the regulation of insulin secretion. Similar expression data are found in the mouse, indicating that islet and retina expression are evolutionarily conserved (data not shown). Using global expression data available from human lymphoblastoid cell lines ${ }^{11}$, we did not find any evidence that rs1387153 (or SNPs in LD with rs1387153) directly influences MTNR1B expression. However, similar analysis in pancreatic islets and retina would be necessary to draw conclusions about the possible effects of the rs1387153 SNP on MTNR1B expression. We note that data about rs1387153 association with
Figure 3 MTNR1B mRNA expression by a panel of human tissues and by human pancreatic islets and beta cells. (a) PCR was done for MTNR1B using cDNAs from a commercial panel of human tissues including diencephalon, retina, pancreas, skeletal muscle, liver, heart, lung and kidney. (b) RT-PCR was done for MTNR1B using commercial mRNAs from human whole brain, small intestine and adipose tissue. (c) PCR was done for MTNR1B using cDNAs (c) PCR was done for MTNRIB using CDNAs
from human donors for pancreatic islets and sorted beta cells. PCR products from diencephalon, retina, pancreas, whole brain, pancreatic islets and beta cells were verified by direct sequencing. Molecular weight markers (M) and control lanes for $\beta$-actin are shown.

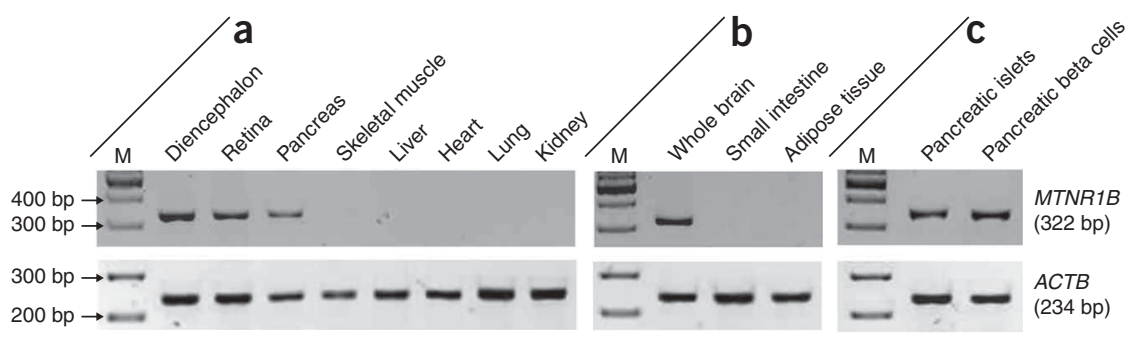

ITN1B $22 \mathrm{bp})$ CTB . . 
MTNR1B expression were not available from a previous human cortical gene expression study ${ }^{12}$.

MTNR1B rs1387153 strongly modulates FPG in the European population $(N=16,094, \beta=0.06 \mathrm{mmol} / \mathrm{l}$ per $\mathrm{T}$ allele, $95 \%$ $\mathrm{CI}=0.05-0.07$; overall $P=7.6 \times 10^{-29}$, unadjusted genome-wide) and increases the risk for T2D. Our findings support a possible link between the regulation of circadian rhythm through melatonin signaling and glucose homeostasis.

\section{METHODS}

Study participants. A GWA meta-analysis for FPG was done in nondiabetic French individuals with European ancestry from our recent genome-wide T2D ${ }^{13}$ and obesity case-control studies (unpublished data). We analyzed GWA data from four populations (Supplementary Table 1) including (i) normal-weight nondiabetic adults selected from the DESIR prospective study ${ }^{14}$ (see below for further details), (ii) normal-weight nondiabetic children from the STANISLAS study ${ }^{15}$ and the Fleurbaix-Laventie Ville Santé II study ${ }^{16}$, (iii) obese nondiabetic adults recruited by the CNRS UMR8090 and the Department of Nutrition of Paris Hotel Dieu hospital and (iv) obese nondiabetic children recruited by the CNRS UMR8090 and the Toulouse Children's Hospital.

The Data from the Epidemiological Study on the Insulin Resistance Syndrome (DESIR) cohort was used for the replication of the association with FPG and metabolic traits, for the incidence of hyperglycemia and the cumulative effect of FPG-associated SNPs (MTNR1B rs1387153, G6PC2 rs560887, GCKR rs1260326 (P446L) and GCK rs1799884 (-30G/A) variants). DESIR is a longitudinal French general population cohort and is fully described elsewhere ${ }^{5,14}$. Because ancestry could not be legally documented at the beginning of the DESIR study, we estimated the proportion of subjects having non-European ancestry among 658 DESIR subjects, using 328 SNPs highly differentiated among individuals from different continents $\left(F_{\mathrm{ST}}>0.2 \text { calculated from the Perlegen dataset }\right)^{17}$ and STRUCTURE software ${ }^{18}$, as described elsewhere ${ }^{5,13}$. We also excluded participants born overseas before analyses in order to minimize hidden population structure in the remaining participants. We analyzed 3,886 subjects who had been successfully genotyped, and who were not overlapping with the GWA data individuals, with FPG available at baseline (Supplementary Table 1). Three classes of FPG status were defined according to the 1997 American Diabetes Association criteria $^{2}$ : normal glucose (NG), defined as FPG $<6.1 \mathrm{mmol} / \mathrm{l}$; impaired fasting glucose (IFG), defined as FPG between 6.1 and $6.99 \mathrm{mmol} / \mathrm{l}$; and T2D, defined as FPG $\geq 7.0 \mathrm{mmol} / \mathrm{l}$ and/or treatment by glucose lowering agents. Hyperglycemia was defined by IFG or T2D. The homeostasis index of insulin release (HOMA-B) was calculated as HOMA-B $=(20 \times$ fasting insulin $) /$ (fasting glucose - 3.5), where fasting insulin is in $\mathrm{mU} / \mathrm{l}$ and fasting glucose in $\mathrm{mmol} / \mathrm{l}$ (ref. 19). We analyzed 3,365 participants from the DESIR cohort who had been successfully genotyped and followed for incident IFG and T2D during a 9-year period, including 515 hyperglycemia incident cases. All the DESIR participants signed an informed consent and the study protocol was approved by the ethics committee for the protection of subjects for biomedical research of the Bicêtre Hospital, Paris, France.

The Inter99 study sample was used for the replication of the association of rs1387153 with FPG and metabolic traits, and has been fully described previously ${ }^{20}$. Glucose tolerance was assessed for 5,877 individuals from the population-based Inter99 study sample who had been successfully genotyped for rs1387153. Subjects with known T2D $(N=114)$ or who were identified as diabetic during the oral glucose tolerance test (OGTT)(FPG $>7 \mathrm{mmol} / \mathrm{l}$ or $120 \mathrm{~min}$ OGTT $>11.1 \mathrm{mmol} / \mathrm{l}, N=245$ individuals) were excluded from the FPG replication analysis and were included in the case-control analysis (see below). The remaining 5,518 nondiabetic individuals were investigated for an association with FPG and metabolic traits (Supplementary Table 1). HOMA-IR and HOMA-B was calculated using the HOMA2-calculator ${ }^{21}$. We tested the Inter99 population for population stratification, applying the program STRUCTURE ${ }^{18}$ on 37 randomly picked SNPs from 37 genes. No evidence of population stratification was found. All participants were of European ancestry and Danish nationality and informed written consent was obtained from all participants before participation. The study was approved by the ethical committees of Copenhagen and was in accordance with the principle of the Helsinki Declaration II.
We used the Northern Finland 1986 Birth Cohort (NFBC86) for the replication of the association of rs1387153 with FPG. NFBC86 is a prospective birth cohort including all Finnish mothers of European ancestry with children whose expected date of birth fell between July 1, 1985 and June 30, 1986 in the two northernmost provinces in Finland ${ }^{22}$. Clinical examination at 15-16 years follow-up was conducted between August 2001 and June 2002. All cohort members living in Finland with known address $(n=9,215)$ were invited, and 6,798 participated (74\%). We analyzed 5,237 nondiabetic subjects successfully genotyped in the NFBC86 cohort with FPG available. The study was carried out in accordance with the latest version of the Declaration of Helsinki. All participants and their parents gave a written informed consent. The study protocol has been approved by the ethics committee of the Faculty of Medicine of the University of Oulu.

We used the Haguenau population for the replication of the association of rs1387153 with FPG. The Haguenau study is a community-based cohort of young adults that investigates long-term consequences of being born small for gestational age and was fully described elsewhere ${ }^{23}$. Briefly, subjects born between 1971 and 1985 were identified from a population-based registry of Haguenau, France. In this area, subjects with non-European ancestry are estimated to comprise less than $0.1 \%$ of the general population ${ }^{23}$. At a mean age of 22 years, participants underwent a medical examination to assess anthropometric and clinical parameters. We analyzed 1,453 nondiabetic subjects successfully genotyped in the Haguenau cohort with FPG available (Supplementary Table 1). The Haguenau study protocol was approved by the ethics committee of ParisSt. Louis University, Paris, France.

Type 2 diabetes cases. We analyzed 4,098 unrelated French individuals with T2D ascertained from the French T2D Family and Obesity Family studies, collected by the CNRS-UMR8090 Unit (Pasteur Institute, Lille) and the Endocrinology-Diabetology Department of the Corbeil-Essonnes Hospital, the Diab2-Néphrogène/Surdiagène $s t u d y^{24}$ or the DESIR study. Details about these cases are provided elsewhere ${ }^{13}$. We used 4,266 normoglycemic $($ FPG $<6.1 \mathrm{mmol} / \mathrm{l})$ DESIR participants as controls. Individuals with T2D of non-European ancestry were excluded after population structure analysis described previously ${ }^{13}$.

The Danish case-control study included all unrelated type 2 diabetes cases and healthy, glucose-tolerant controls with successful genotyping from the Inter99 study sample (cases $=339$, control individuals $=4,368$ ) and individuals recruited from the outpatient clinic at Steno Diabetes Center (cases $=$ 1,895 , control individuals $=498$ ). Individuals with T2D with non-European ancestry were excluded before analysis.

Genotyping. GWA genotypes were obtained using the Illumina Human CNV370-Duo array (unpublished data). Genotyping was done using $750 \mathrm{ng}$ of genomic DNA following the manufacturer's protocols (Illumina). In addition, 523 French lean adult controls previously genotyped using the Illumina HAP300 array $^{13}$ were included in the analysis. We used the Illumina's BeadStation genotyping solutions, based on the GenCall software application, to automatically cluster, call genotypes, and assign confidence scores. The GenCall application incorporates a clustering algorithm (GenTrain) and a calling algorithm. Genotyping calls for a specific DNA are made by the calling algorithm, relying on information provided by the GenTrain clustering algorithm. We identified 311,398 SNPs shared by the two arrays; of these, we discarded 2,552 SNPs that had extreme Hardy-Weinberg disequilibrium in the control samples $(P<0.001)$, low genotyping calling rates $(<95 \%)$ or low minor-allele frequencies $(<1 \%)$. We retained 308,846 SNPs for analysis. Call rates in the CNV370-Duo and the HAP300 arrays were 99.6\% and 99.7\%, respectively. Two individuals were genotyped twice using the Illumina Human CNV370-Duo array and the concordance rate was $99.75 \%$. Genomic control $\lambda_{\mathrm{GC}}$ was $1.00,1.01,1.00$ and 1.00 for control adults, control children, obese adults and obese children, respectively.

We used TaqMan assays to genotype all replication populations. These were designed and carried out according to the manufacturer's instructions (AB assay numbers c-1932612-10 and c-27978210-10 for rs1387153 and rs4240702, respectively). Call rate was at least $98 \%$ and no significant deviation $(P>0.1)$ from Hardy-Weinberg equilibrium was observed in the populations studied. 
Sequencing. The MTNR1B gene encodes a 362-amino-acid protein (MIM600804). The two exons of MTNR1B were analyzed in five fragments. Primer sequences and PCR conditions are available in Supplementary Table 6. Fragments were sequenced using a standard protocol and the automated 3730xl DNA Analyzer (Applied Biosystems). Electrophoregram reads were assembled and analyzed with the Variant Reporter software (Applied Biosystems).

Expression analyses. For PCR amplification of CDNAs, we used commercial cDNAs from the Human MTC panel I (BD Biosciences Clontech) and the Human Adult Normal Tissue for retina and diencephalon (BioChain Institute). cDNA samples were amplified by standard PCR using the Fast Start Taq (Roche Applied Science). All reactions were run for 45 cycles. Primer sequences and PCR conditions are detailed in the Supplementary Table 6. Each expression was assessed in duplicates. The PCR products were electrophoresed on a 3\% agarose gel and visualised by ethidium bromide staining exposed to UV light.

For the cDNA reverse transcription analyses, we used RNA extracted from the whole brain, small intestine and adipose tissue, which we obtained from the Human Adult Normal 5 Donor Pool (BioChain Institute). cDNA synthesis by reverse transcription was done using the MultiScribe Reverse Transcriptase and random primers according to the manufacturer's instructions (Applied Biosystems).

We isolated pancreatic islets of Langerhans and sorted beta cells from adult brain-dead donors in accordance with the French regulations and with the local institutional ethical committee, as previously described ${ }^{25}$. Briefly, pancreatic islets were isolated after ductal distension of the pancreata and digestion of the tissue with Liberase (Roche Diagnostics). Human beta cells were sorted by FACS analysis of semi-purified preparations of islet cells using Newport Green, a specific zinc-fluorescent probe ${ }^{25}$.

Statistical analyses. We carried out GWA analysis using linear regression models to test the association between FPG and genome-wide data, with adjustment for age, gender and BMI in each subgroup, using PLINK ${ }^{26}$. The summary statistics were combined in the meta-analysis using the inverse normal method with equal weight for each population. In this method, $P$ values of each study are transformed into their inverse normal $z$ score and the weighted sum, over all studies, is compared to a normal $\mathrm{N}(0,1)$, provided the sum of squared weights equals 1 . Bonferroni-adjusted $P$ values were obtained as $P$ value $\times 308,846$. SNPs available from HapMap but not directly genotyped on the Illumina $370 \mathrm{~K}$ mapping array were imputed (mean posterior probability $>0.91$ ) using IMPUTE to study a region extending $100-\mathrm{kb}$ on each side of rs1387153. Association of these imputed SNPs with FPG levels was assessed using SNPTEST ${ }^{27,28}$, which uses a likelihood framework to account for the uncertainty of genotype calling. In the replication studies, we analyzed the effect of rs1387153 on FPG in nondiabetic individuals (FPG $<7 \mathrm{mmol} / \mathrm{l}$ ) using linear regression models. The estimates of the rs 1387153 effect on FPG and their standard errors for each separate analysis were combined in the metaanalysis using the weighted inverse normal method, and the overall effect and its confidence interval were estimated using the inverse variance method implemented in 'meta.summaries' function of R RMETA package. No major heterogeneity in effects was observed $(P=0.02)$ and when the Haguenau study was removed, the $P$ value for heterogeneity went up to 0.05 . When including the discovery cohorts into the overall meta-analysis, we used weights equal to the sizes of the populations, because of an important difference in sample sizes (for example, 22-fold between obese adults and NFBC86). The $P$ value for heterogeneity was 0.09 . The effect of rs 1387153 on diabetic status was tested using a logistic regression model using the $\mathrm{R}$ mgcv package, adjusted for age, sex and BMI. In the DESIR participants, we assessed the effect of rs 1387153 on hyperglycemia incidence with Cox proportional-hazards regression models, adjusted for sex and BMI. Survival time was age at diagnosis or censoring. All statistical analyses of the replication data were done with $\mathrm{R}$ (version 2.6.1), combined with the survival, rmeta and mgcr packages.

URLs. DIAGRAM data, http://www.well.ox.ac.uk/DIAGRAM/meta.html; conservation across species data, http://www.bx.psu.edu/miller_lab/; eQTls data from human lymphoblasts study, http://www.sph.umich.edu/csg/liang/asthma/; eQTL data from the human cortical gene expression study, http://labs.med. miami.edu/myers/; Homa2 calculator, www.dtu.ox.ac.uk/homa.
Note: Supplementary information is available on the Nature Genetics website.

\section{ACKNOWLEDGMENTS}

The study was supported in part by an ALFEDIAM-Les laboratoires Servier grant, the "Conseil Regional Nord-Pas-de-Calais: Fonds européen de développement économique et regional," Genome Quebec-Genome Canada and the British Medical Research Council. N.B.-N.'s position is supported by a grant from the ANR (Agence Nationale pour la Recherche: ANR-06 PHYSIO - 037 -02). A.B. is funded by a research fellowship from the French nonprofit "Association pour l'Etude des Anomalies Congénitales." We acknowledge funding to P.F. by the European Union (Integrated Project EURODIA LSHM-CT-2006-518153 in the Framework Programme 6 [FP06] of the European-Community). We thank M. Deweirder and F. Allegaert for DNA extraction of part of the cohorts studied; S. Gaget and S. Gallina for bioinformatics support; S. Poulain and P. Gallina for the recruitment of obese children families; B. Guardiola-Lemaitre for fruitful discussion on results; and C. Lecoeur for statistical assistance. We acknowledge the "Centre de Médecine Préventive (CMP) de Vandoeuvre-Les-Nancy" where the STANISLAS cohort was recruited. The DESIR study has been supported by INSERM, CNAMTS, Lilly, Novartis Pharma and Sanofi-Aventis, the Association Diabète Risque Vasculaire, the Fédération Française de Cardiologie, La Fondation de France, ALFEDIAM, ONIVINS, Ardix Medical, Bayer Diagnostics, Becton Dickinson, Cardionics, Merck Santé, Novo Nordisk, Pierre Fabre, Roche and Topcon. The Diab-2-Néphrogène/Surdiagène study acknowledges the participating patients, physicians and the staff of the CIC Poitiers, PHRC (Projet Hospitalier de Recherche Clinique), and a 2003-AFD-grant. The NFBC86 is supported by the European Commission; contract number QLG1-CT-2000-01643, Biocenter, University of Oulu, Finland and the Academy of Finland. We thank L. Peltonen for providing NFBC86 DNA samples. The Inter99 study was supported by grants from the Lundbeck Foundation Centre of Applied Medical Genomics for Personalized Disease Prediction, Prevention and Care (LUCAMP), the Danish Medical Research Council, Novo Nordisk, the FOOD Study Group/the Danish Ministry of Food, Agriculture and Fisheries, the Danish Diabetes Association and the European Union (EUGENE2, grant no. LSHM-CT-2004-512013), and from the Swedish Research Council (J.H.).

\section{AUTHOR CONTRIBUTIONS}

N.B.-N. was responsible for the design and data analyses, the study follow-up and the manuscript writing. A.B. was responsible for and performed the gene expression study and was involved in the genotyping of the replication stage, the gene sequencing and the manuscript writing. C.C.-P. performed the statistical analysis in the French and Finnish populations and was involved in the manuscript writing. T.S. and J.H. performed statistical analysis in the Danish samples and were involved in the manuscript writing. M. Marchand was involved in the genotyping of the replication stage, gene expression and gene sequencing. J.D. supervised the genotyping of the GWA data. S.L. and E.D. performed the GWA genotyping. G.R. and R.S. were involved in the type 2 diabetes and obesity GWA studies and the manuscript writing. F.D.G. was involved in the bioinformatics analyses for the GWA studies and the in silico eQTL analyses. J.-C.C. performed the in silico eQTL analyses. K.B.-J., A.-L.H., A.R., J.T., M. Marre, J.W., B.H., M.T., P.E., T.J., G.C., S.H., S.V.-S. and C.L.-M. provided DNA samples and phenotype data. B.B. and M.-R.J. provided DNA samples and phenotype data and were involved in the manuscript writing. T.H. and O.P. provided access to DNA samples and phenotype data, supervised the genotyping and the statistical analyses in the Danish samples and were involved in the manuscript writing. D.M. supervised the obesity GWA study. S.C. and M.V. were involved in the manuscript writing. F.P. provided the human pancreatic islets and sorted beta cells. K.L. and F.S. provided expression data in the mouse. A.I.F.B. and A.J.W. were involved in the obesity GWA study and the manuscript writing. C.D. was involved in the study design and the manuscript writing and supervised the statistical analyses. P.F. was the principal investigator of the study and was involved in the study design and the manuscript writing. All authors approved the data and the final manuscript.

Published online at http://www.nature.com/naturegenetics/

Reprints and permissions information is available online at http://npg.nature.com/ reprintsandpermissions/

1. American Diabetes Association. Standards of medical care in diabetes-2008. Diabetes Care 31 (Suppl. 1), S12-S54 (2008).

2. The Expert Committee on the Diagnosis and Classification of Diabetes Mellitus. Report of the Expert Committee on the Diagnosis and Classification of Diabetes Mellitus. Diabetes Care 20, 1183-1197 (1997)

3. Tirosh, A. et al. Normal fasting plasma glucose levels and type 2 diabetes in young men. N. Engl. J. Med. 353, 1454-1462 (2005). 
4. Barr, E.L. et al. Risk of cardiovascular and all-cause mortality in individuals with diabetes mellitus, impaired fasting glucose, and impaired glucose tolerance: the Australian Diabetes, Obesity, and Lifestyle Study (AusDiab). Circulation 116, 151-157 (2007).

5. Bouatia-Naji, N. et al. A polymorphism within the G6PC2 gene is associated with fasting plasma glucose levels. Science 320, 1085-1088 (2008).

6. Peschke, E. Melatonin, endocrine pancreas and diabetes. J. Pineal Res. 44, 26-40 (2008).

7. Van Cauter, E. Putative roles of melatonin in glucose regulation. Therapie $53,467-472$ (1998).

8. Boden, G., Ruiz, J., Urbain, J.L. \& Chen, X. Evidence for a circadian rhythm of insulin secretion. Am. J. Physiol. 271, E246-E252 (1996).

9. Peschke, E. et al. Diabetic Goto Kakizaki rats as well as type 2 diabetic patients show a decreased diurnal serum melatonin level and an increased pancreatic melatoninreceptor status. J. Pineal Res. 40, 135-143 (2006).

10. Ramracheya, R.D. et al. Function and expression of melatonin receptors on human pancreatic islets. J. Pineal Res. 44, 273-279 (2008).

11. Dixon, A.L. et al. A genome-wide association study of global gene expression. Nat. Genet. 39, 1202-1207 (2007).

12. Myers, A.J. et al. A survey of genetic human cortical gene expression. Nat. Genet. 39 1494-1499 (2007).

13. Sladek, R. et al. A genome-wide association study identifies novel risk loci for type 2 diabetes. Nature 445, 881-885 (2007).

14. Balkau, B. An epidemiologic survey from a network of French Health Examination Centres, (D.E.S.I.R.): epidemiologic data on the insulin resistance syndrome. Rev. Epidemiol. Sante Publique 44, 373-375 (1996).

15. Visvikis-Siest, S. \& Siest, G. The STANISLAS Cohort: a 10-year follow-up of supposed healthy families. Gene-environment interactions, reference values and evaluation of biomarkers in prevention of cardiovascular diseases. Clin. Chem. Lab. Med. 46, 733-747 (2008).

16. Heude, B. et al. Anthropometric relationships between parents and children throughout childhood: the Fleurbaix-Laventie Ville Sante Study. Int. J. Obes. (Lond) 29, 1222-1229 (2005).
17. Hinds, D.A. et al. Whole-genome patterns of common DNA variation in three human populations. Science 307, 1072-1079 (2005).

18. Pritchard, J.K., Stephens, M. \& Donnelly, P. Inference of population structure using multilocus genotype data. Genetics 155, 945-959 (2000).

19. Matthews, D.R. et al. Homeostasis model assessment: insulin resistance and beta-cell function from fasting plasma glucose and insulin concentrations in man. Diabetologia 28, 412-419 (1985).

20. Jorgensen, T. et al. A randomized non-pharmacological intervention study for prevention of ischaemic heart disease: baseline results Inter99. Eur. J. Cardiovasc. Prev. Rehabil. 10, 377-386 (2003).

21. Wallace, T.M., Levy, J.C. \& Matthews, D.R. Use and abuse of HOMA modeling. Diabetes Care 27, 1487-1495 (2004).

22. Jarvelin, M.R. et al. Ecological and individual predictors of birthweight in a northern Finland birth cohort 1986. Paediatr. Perinat. Epidemiol. 11, 298-312 (1997).

23. Jaquet, D., Collin, D., Levy-Marchal, C. \& Czernichow, P. Adult height distribution in subjects born small for gestational age. Horm. Res. 62, 92-96 (2004).

24. Hadjadj, S. et al. Prognostic value of the insertion/deletion polymorphism of the ACE gene in type 2 diabetic subjects: results from the Non-insulin-dependent Diabetes, Hypertension, Microalbuminuria or Proteinuria, Cardiovascular Events, and Ramipril (DIABHYCAR), Diabete de type 2, Nephropathie et Genetique (DIAB2NEPHROGENE), and Survie, Diabete de type 2 et Genetique (SURDIAGENE) studies. Diabetes Care 31, 1847-1852 (2008).

25. Lukowiak, B. et al. Identification and purification of functional human beta-cells by a new specific zinc-fluorescent probe. J. Histochem. Cytochem. 49, 519-528 (2001).

26. Purcell, S. et al. PLINK: a tool set for whole-genome association and population-based linkage analyses. Am. J. Hum. Genet. 81, 559-575 (2007).

27. Burton, P.R. et al. Association scan of 14,500 nonsynonymous SNPs in four diseases identifies autoimmunity variants. Nat. Genet. 39, 1329-1337 (2007).

28. Marchini, J., Howie, B., Myers, S., McVean, G. \& Donnelly, P. A new multipoint method for genome-wide association studies by imputation of genotypes. Nat. Genet. 39, 906-913 (2007). 